\title{
Equilibrium Analysis in Wireless Networks Walrasian Markets: A Distributed Approach
}

\author{
Vahid Haghighatdoost*, Siavash Khorsandi*, Zaheer Khan ${ }^{\dagger}$, Hamed Ahmadi ${ }^{\ddagger}$ \\ * Computer Engineering and IT Department, Amirkabir University of Technology, Tehran, Iran. \\ $\dagger$ The 6G Flagship centre, University of Oulu, Finland. \\ $\ddagger$ Department of Electronic Engineering, University of York, United Kingdom.
}

\begin{abstract}
A Walrasian Market can be modeled as a distributed system consisting a set of independent buyers and sellers. The Walrasian equilibrium theorem proves the existence of the optimal price that results in the market clean state or Walrasian Equilibrium where the sum of absolute excess demand is zero. It is proved that finding this equilibrium price is an NP-hard problem. In this paper, we present an efficient distributed controltheoretic approach for finding the Walrasian equilibrium in an exchange economy. We have modeled the price adjustment process as a closed-loop control system where the sum of absolute excess demand is measured as the system error that is fed to commodity moderators in a distributed schema simultaneously, and then each commodity moderator adjusts the price of its related commodity. We devised a controller algorithm with low complexity and fast convergence that iteratively moves the error value to zero. The proposed scheme, finds the equilibrium price and Pareto efficient allocation without knowing the shape of user utility functions or their preferences. It is scalable and is usable for exchange economies with multiple goods and many types of users.
\end{abstract}

Index Terms-Walrasian equilibrium, Dynamic Spectrum Allocation, Pareto efficient allocation, Price adjustment

\section{INTRODUCTION}

A Walrasian Market is formed by a number of independent commodity providers in a competitive setting that are serving possibly numerous independent buyers both acting in rationally selfish manner. The clean state market is the condition where the total demand equals the total supply. Since the demand is function of the commodity price, in clean state market, the optimal price $P^{*}$ is calculated by commodity providers in a distributed manner where there would be no available excess budget or commodity. Finding the equilibrium condition in a fully competitive market is a well-known problem. The existence of an equilibrium in these markets has already been proven and has been a well-recognized theorem [1], [2], [3]. However, finding the equilibrium allocations and corresponding prices is an NP-hard problem [4]. There have been many attempts to propose efficient solutions for various categories of problems. In [5], the authors offer a solution for the case where the utility functions are linear. In [6], the authors developed an approximate scheme based on reducing the calculation of a Walrasian equilibrium to a polynomial number of concave maximizations. It involves construction of simple subdivisions and some form of exhaustive search in the labeling process and the results may not be very accurate. The authors of [7] investigate a computable general equilib- rium (CGE) modeling theory and provide a general algebraic framework including numerical equations to be solved for the equilibrium values of economic variables. However, it does not provide a detailed solution or guidelines.

Developing algorithms to reach a fair market equilibrium has been a point of interest in many fields and in particular in the context of next generation wireless service networks [8], [9], [10], [11]. Walrasian equilibrium governed by the laws of demand and supply is of particular interest. There are a number of previous attempts to develop theories and algorithms to find or move the system towards a fair Walrasian equilibrium [4], [6], [12]. None of these works, however, produced a general numerically sound approach to solve the Walrasian equilibrium problem. In [8], load balancing in a heterogeneous wireless access networks (HWAN) is considered. A 'heterogeneous handoff management system' is proposed where three sub-problems, base station (BS) and radio access technology (RAT) by mobile terminals and 'weight restriction' technique used by the base stations, are independently solved and it is shown that applying appropriate weight restriction technique results in a balanced equilibrium condition. In [9], an autonomous connection management (ACM) mechanism is developed to strike a balance between the overall system throughput, load distribution in the network, fairness in access to resources and user satisfaction in in HWAN settings. A stochastic equilibrium analysis framework was devised that showed favorable system conditions under various scenarios. In [10], authors modeled the system dynamics as a novel three-stage game creating a unified framework for spectrum allocation, network best response and user welfare. In this work, frequency spectrum was considered as a commodity and model the spectrum allocation was modeled as a market dynamics problem. System equilibrium was analyzed by finding the Nash equilibrium of the game through which the influence of regulatory decisions and impact of network/user strategies is analyzed. The devised model provides a framework for the regulatory and network operators for adjusting spectrum allocation table towards maximizing the social welfare for all the players. In [13], the economics of spectrum sharing, which is referred to as spectrum trading, is addressed in cognitive radio networks. The authors introduced a market-equilibriumbased spectrum trading mechanism that uses spectrum demand and supply of the primary and secondary users, respectively. Since spectrum supply is stochastic in nature, a distributed 
and adaptive learning algorithm was proposed to be used for the secondary users to estimate spectrum price and adjust the spectrum demand accordingly so that a fair market equilibrium can be reached.

In this paper, we propose an iterative process based on the theory of closed-loop control system that conveniently converges to the Walrasian equilibrium. Stability and convergence of the proposed algorithm is demonstrated through simulations. Our main contributions in this work are as follows:

- We propose a numerical solution to find Walrasin equilibrium without any assumption on the format of clients' utility functions; it is only required that the clients' utility functions be quasi-concave;

- Scalability of the model in terms of the number of clients is established through numerical results;

- Stability of the proposed approach to find the Walrasian equilibrium is guaranteed as the sum on absolute excess demand progressively converges to zero.

The rest of this paper is organized as follows. In Section 2, we formally state the principles of Walrasian equilibrium in an exchange economy. In Section 3, we introduce the proposed control-theoretic algorithm to find the Walrasian economy. In Section 4, the convergence and stability of the proposed algorithm is analyzed. Section 5 contains a case study, applying the proposed algorithm in a dynamic spectrum allocation problem as a sample application area and finally Section 6 contains the conclusion and final remarks.

\section{EXCHANGE ECONOMY AND WALRASIAN EQUILIBRIUM}

Let us consider an exchange market where different sellers and buyers are to exchange their commodities. In a clean market state, all commodities are expected to be fully assigned. There is an equilibrium price for each commodity at this state. In addition, allocation of commodities to each client is considered to be Pareto efficient [1]. That means, there is no way to make someone better off without making someone else worse off.

Consider the economy $E$ where we have $N$ commodities and $M$ players, $\mathcal{J}=\{1, \ldots, N\}$ is the index set of commodities while $\mathcal{I}=\{1, \ldots, M\}$ is the index set of the market players called clients here and that every client is endowed with a non-negative amount of each commodity as its initial asset. Client's commodity preferences is defined on the consumption set $R_{+}^{N}$, denoting positive real numbers, and their utilities are represented by a utility function $u^{i}$ fulfilling some set properties.

In the competitive exchange process, all clients know about the prices of all commodities, and they act as both a buyer and a seller; i.e., a client can sell her goods to buy goods from other clients. Mapping this to a real market, money can be also considered as a special commodity where the sellers are only interested in this type of commodity.

Suppose $\boldsymbol{p}=\left(p_{1}, \ldots, p_{N}\right) \gg 0$ is the vector of prices for $N$ available commodities, and $\boldsymbol{e}^{i}=\left(e_{1}^{i}, \ldots, e_{N}^{i}\right)$ is the initial asset or initial endowment of client $i$. The vector $\boldsymbol{x}^{\boldsymbol{i}}=$ $\left(x_{1}^{i}, \ldots, x_{N}^{i}\right)$ denotes the amounts of client $i$ from $N$ different commodities after redistribution of commodities that we call it as "demand allocation vector". Clients try to increase their payoff through the exchange process simultaneously, which is mathematically defined as follows:

$$
\begin{gathered}
\max u^{i}\left(\boldsymbol{x}^{\boldsymbol{i}}\right) \\
\text { subject to: } \boldsymbol{p} \cdot \boldsymbol{x}^{i} \leqslant \boldsymbol{p} \cdot \boldsymbol{e}^{\boldsymbol{i}} \\
\boldsymbol{x}^{\boldsymbol{i}} \in R_{+}^{N}
\end{gathered}
$$

The factor $\boldsymbol{p} \cdot \boldsymbol{e}^{\boldsymbol{i}}$ is a constant value in the optimization problem that determines the client's budget. Therefore, (1b) demonstrates the client's budget constraint and limits the client's purchase power to its total budget. The outcome of this optimization problem solved independently by each client $i$ is $\chi^{i}(\boldsymbol{p})$ which represents the best and most favorable request bundle for each client and depends on the current price vector and the client's initial asset $\left(\boldsymbol{x}^{i}=\chi^{i}(\boldsymbol{p})\right)$. However, the supply limitation is not enforced, i.e. commodity availability is not considered. Hence, the overall demand for some commodities may excess the available supply. To formulate supply surplus or shortage, we define the aggregate excess demand for commodity $j$ by $z_{j}$ :

$$
z_{j}=\sum_{i=1}^{M} x_{j}^{i}-\sum_{i=1}^{M} e_{j}^{i} .
$$

Here, $e_{j}^{i}$ denotes the amount of commodity $j$ that client $i$ has and $x_{j}^{i}$ denotes the amount of commodity $j$ that client $i$ desires to have. As $\boldsymbol{x}^{\boldsymbol{i}}$ is a function of price $\boldsymbol{p},\left(\boldsymbol{x}^{\boldsymbol{i}}=\chi^{i}(\boldsymbol{p})\right)$, in this regard, we can define $z_{j}$ as a function of price $z_{j}=z_{j}(\boldsymbol{p})$.

There is excess demand for commodity $j$ when $z_{j}(\boldsymbol{p})>0$, and there is excess supply of this commodity when $z_{j}(\boldsymbol{p})<0$. Thus, $\boldsymbol{z}(\boldsymbol{p})=\left(z_{1}(\boldsymbol{p}), \ldots, z_{N}(\boldsymbol{p})\right)$ is defined as the aggregate excess demand vector as a function of price.

\section{A. Principles of exchange economies}

In the following, we describe the basic principles that apply in an exchange economy that forms the basis for our proposed algorithm explained in the next section.

1) Clients' utility specification: Utility function $u^{i}$ for client $i$, should be continuous, strongly increasing, and strictly quasi-concave on $R_{+}^{N}$ [1].

2) Existence and uniqueness of the demand allocation vector: For every price vector $\boldsymbol{p}$, we have a unique solution, $\mathbf{X}=\left[\boldsymbol{x}^{\mathbf{1}}, . ., \boldsymbol{x}^{\boldsymbol{M}}\right]$, to the optimization problem in (1) which represents the most favorable request bundle for the clients under current commodity prices and clients' purchase powers [1]. This means the exchange process has a unique answer for each price vector where the utilities of all clients would be at their maximum possible values if there were no supply limitations. 


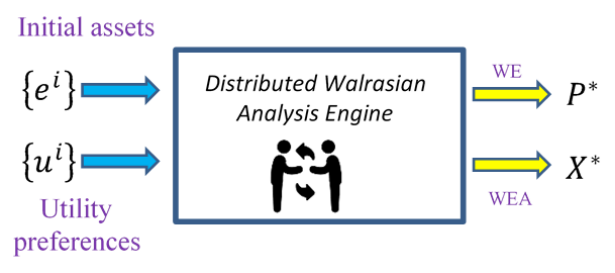

Fig. 1: A system-level view of Walrasian Analysis engine

3) Walrasian Equilibrium (WE): A price vector $\boldsymbol{p}^{*} \gg \mathbf{0}$ is called a Walrasian equilibrium if $\boldsymbol{z}\left(\boldsymbol{p}^{*}\right)=\mathbf{0}$ [1]. If such a price vector exists, the sum of redistributions for a commodity $j$ is equal to its total initial supply by all clients:

$$
\forall j \in\{1, \ldots, N\}, \sum_{i=1}^{M} x_{j}^{i}(\boldsymbol{p})=\sum_{i=1}^{M} e_{j}^{i} .
$$

Let $p^{*}$ be a Walrasian equilibrium for some economy with initial endowments $\mathbf{e}$, then $\mathbf{X}^{*}=\left(\chi^{1}\left(\boldsymbol{p}^{*}\right), \ldots, \chi^{M}\left(\boldsymbol{p}^{*}\right)\right)$ is called a Walrasian equilibrium allocation, or WEA.

4) Existence of the clean market price: Assuming principle II-A3 and given $\sum_{i=1}^{M} e^{i} \gg \mathbf{0}$, then there exists at least one price vector, $\boldsymbol{p}^{*} \gg \mathbf{0}$, such that $\boldsymbol{z}\left(\boldsymbol{p}^{*}\right)=\mathbf{0}$ [1]. This claims the existence of an equilibrium price vector $p^{*} \in R_{+}^{N}$ that moves the excess demand vector to its optimal state $\left(\boldsymbol{z}\left(\boldsymbol{p}^{*}\right)=\mathbf{0}\right)$.

5) First Welfare Theorem: Every Walrasian equilibrium allocation is Pareto efficient [1]. Therefore, there is no way to increase the payoff of one client without decreasing at least one other client.

6) Second Welfare Theorem: Any price vector $\tilde{\boldsymbol{p}}$ that makes the excess demand zero $(\boldsymbol{z}(\tilde{\boldsymbol{p}})=\mathbf{0})$ is a Walrasian equilibrium $\left(\tilde{\boldsymbol{p}}=\boldsymbol{p}^{*}\right)$, and the related allocation is WEA [1].

These principles establish the existence of the Walrasian equilibrium and the related necessary conditions. However, obtaining an actual solution is a daunting task and remains to be a research issue. In the following, we endeavor to develop a practical solution to find a price vector that results in zero excess demand.

\section{Proposed Walrasian Analysis Engine}

A conceptual view of the Walrasian analysis engine is shown in Figure 1. The initial assets of clients and their utility preferences that satisfies Principle 1 of exchange economies act as inputs. Through interactions between the sellers and buyers' the price and demand vectors are adjusted independently in a selfish manner. The final outcome of the system is the optimal price and optimal allocations $\boldsymbol{p}^{*}$ and $\mathbf{X}^{*}$ that results in clean-state condition.

We can consider the Walrasian analysis engine as a closedloop control system with feedback defined by iteratively diminishing the error signal, $\boldsymbol{z}(\boldsymbol{p})$, its outputs converge to the $\boldsymbol{p}^{*}$ and $\mathrm{X}^{*}$. The Walrasian analysis engine involves three sub parts: client behavior simulator, market behavior simulator and price adjustment feedback control box (Figure 2). Market behavior is simulated based on optimization process defined in

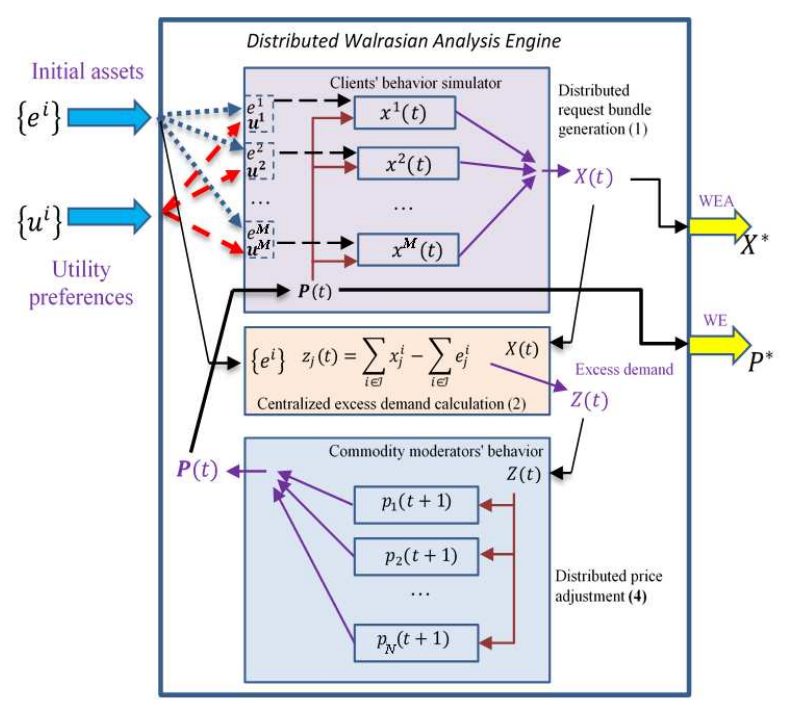

Fig. 2: Sub parts of Walrasian Analysis engine.

(1). The feedback control algorithm used at price adjustment box is the heart of the proposed approach that implements the price adjustment process.

At each adjustment cycle $t$, the optimization problem in (1) is solved by client entities. In our implementation, we use CVX [14] optimization package to solve (1) to model clients' behavior as a convex optimization. By determining all $x_{j}^{i}(\boldsymbol{p}), \forall i, j$ in this phase, a central entity receives the $x_{j}^{i}$ signals from all clients to calculate the aggregate excess demand for each commodity $j$ using equation (2). To arrive at Walrasian equilibrium $\boldsymbol{p}^{*}$, the system needs to go through the price adjustment process. At each iteration t, we adjust the price in such a way that the excess demand to eventually be zero. Hence, the key point in pricing is that the price for commodities with positive excess demand should increase and the price for commodities with negative excess demand must decrease. We propose the following adjustment policy that demonstrates favorable convergence properties with low computational complexity for $j \in\{1, \ldots, N\}$ :

$p_{j}(t+1)=p_{j}(t)+\frac{\left|z_{j}\right|}{1+\sum_{j=1}^{N}\left|z_{j}\right|}\left(\frac{p_{j}(t) \sum_{i=1}^{M} x_{j}^{i}}{\sum_{i=1}^{M} e_{j}^{i}}-p_{j}(t)\right)$,

where $\sum_{i=1}^{M} x_{j}^{i}$ is total requested amount of commodity $j$ at time $t$. The term $\frac{\left|z_{j}\right|}{1+\sum_{j=1}^{N}\left|z_{j}\right|}$ controls the rate of price adjustment. The price for commodities with higher error value $\left|z_{j}\right|$ are updated with higher rate. This means that the gain of the feedback loop is dynamically adjusted for faster convergence. The term $\frac{p_{j}(t) \sum_{i=1}^{M} x_{j}^{i}}{\sum_{i=1}^{M} e_{j}^{i}}$ semantically determines the optimal price for commodity $j$ where $p_{j}(t) \sum_{i=1}^{M} x_{j}^{i}$ is the total market budget clients are ready to be spent for commodity $j$. This is divided to total amount of supply of this commodity. This amounts to normalizing the error value 


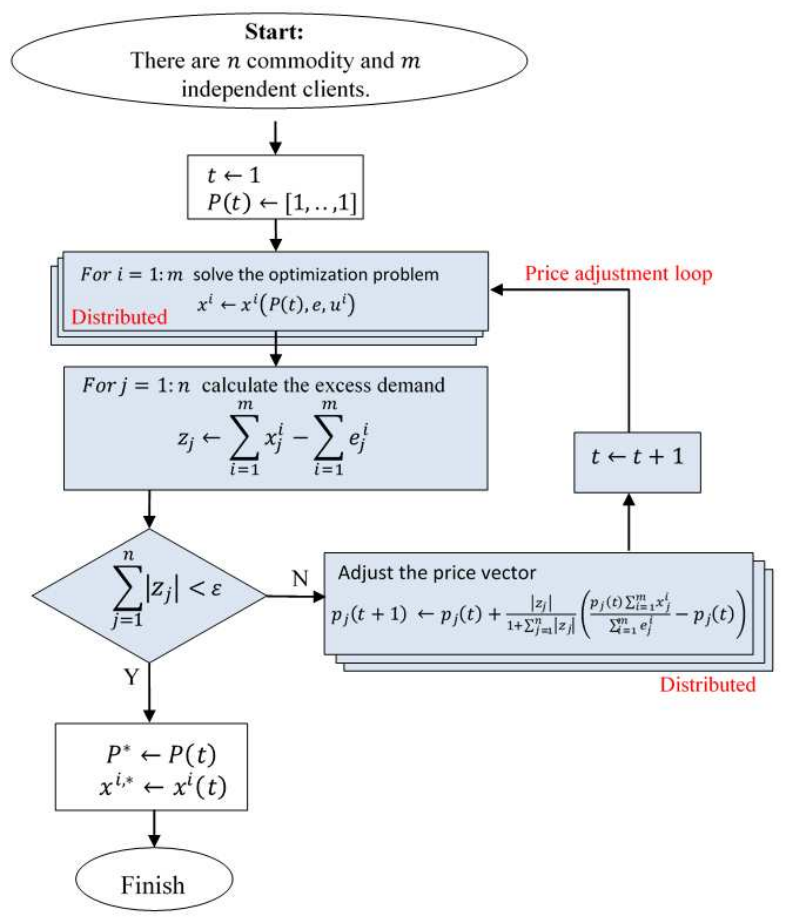

Fig. 3: Flowchart of the proposed approach for finding the Walrasian equilibrium.

TABLE I: Three defined datasets

\begin{tabular}{|l|l|l|l|}
\hline$\#$ & $M$ & $N$ & Amount of Initial Asset $\left(E_{j}=\sum_{i=1}^{M} e_{j}^{i}\right)$ \\
\hline 1 & 50 & 3 & $\boldsymbol{E}=[185.60,47.52,171.85]$ \\
\hline 2 & 100 & 5 & $\boldsymbol{E}=[206.93,63.79,223.72,62.15,191.52]$ \\
\hline 3 & 500 & 10 & $\boldsymbol{E}=[490,168,490,172,502,159,500,173,500,167]$ \\
\hline
\end{tabular}

proportional to the current price of the commodity. The term $\left(\frac{p_{j}(t) \sum_{i=1}^{M} x_{j}^{i}}{\sum_{i=1}^{M} e_{j}^{i}}-p_{j}(t)\right)$ is the error term that decreases through system iterations.

The iterations stops when we meet the market clean state at time $\tau$, at which case, the excess demand converges to zero and we have $\sum_{i=1}^{M} x_{j}^{i}=\sum_{i=1}^{M} e_{j}^{i}$ for all $j \in \mathcal{J}$. At this case, the price vector converges to $\boldsymbol{p}^{*}\left(\forall t>\tau ; p_{j}(t)=p_{j}(\tau)=p_{j}^{*}\right)$. The overall flowchart of the proposed algorithm to find the Walrasian equilibrium is shown in Figure 3. Using defined notation Walrasian $\epsilon$-equilibrium in [6], we defined an adjustable threshold, $\epsilon$, for stopping the iterations which describes the acceptable level of the residual error. Formally, a Walrasian $\epsilon$-equilibrium is a pair $(\boldsymbol{p}, \mathbf{X})$ where $\boldsymbol{p} \in R_{+}^{N}$ and $\mathbf{X}$ is an allocation, we have $\sum_{j=1}^{N}\left|\sum_{i=1}^{M} x_{j}^{i}(\boldsymbol{p})-\sum_{i=1}^{M} e_{j}^{i}\right|<\epsilon$ or $\sum_{j=1}^{N}\left|z_{j}\right|<\epsilon$.

\section{Simulation Results}

To evaluate the proposed feedback based approach, we generated some random datasets as reflected in Table I.

In our simulations, without loss of generality, we considered the Constant Elasticity of Substitution (CES) utility function as the following for each client:

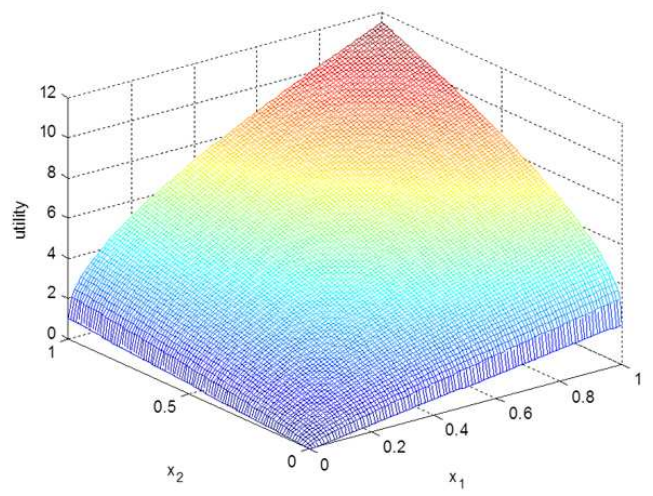

(a)

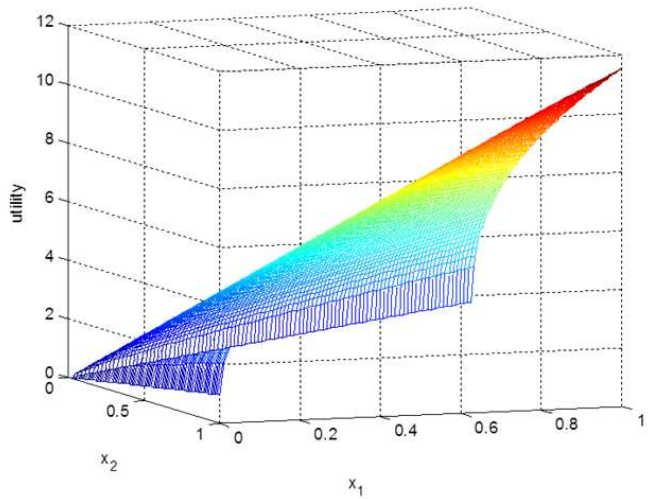

(b)

Fig. 4: Utility function curve for a client in an exchange market with two commodities: $u(\boldsymbol{x})=\left(\sqrt[3]{2 x_{1}}+\sqrt[3]{x_{2}}\right)^{3}$.

$$
u^{i}\left(\boldsymbol{x}^{i}\right)=\left(\sum_{j=1}^{N} \sqrt[r]{w_{j}^{i} x_{j}^{i}}\right)^{r} \forall i \in 1, \ldots, M,
$$

CES is one of the most popular utility functions, which is widely used in micro economic theories and is a suitable model for natural behavior of consumers. The CES utility functions are increasing and concave for $r>1$. The parameter $w_{j}^{i}$ determines the weight of commodity $j$ from client $i$ 's point of view. Figure 4 shows the utility surface for a client in an exchange market with two commodities. We can find that the utility surface is strictly concave.

The initial asset and weight vectors for clients are generated randomly and we set $r=3$ for utility functions and used the threshold $\epsilon=0.5$. The result of applying the proposed algorithm to find the Walrasian equilibrium is depicted in Figure 5.

As depicted in Figure 5, the total market utility increases from the initial level after the exchange process in all cases. Also the Walrasian equilibrium $\left(\boldsymbol{p}^{*}\right)$ for each dataset is presented for each case. The system convergence shows favorable behavior rapidly converging to optimal condition. Using Lyapunov stability theory [15], the convergence of the system can be readily proved as the first derivative of system's potential 


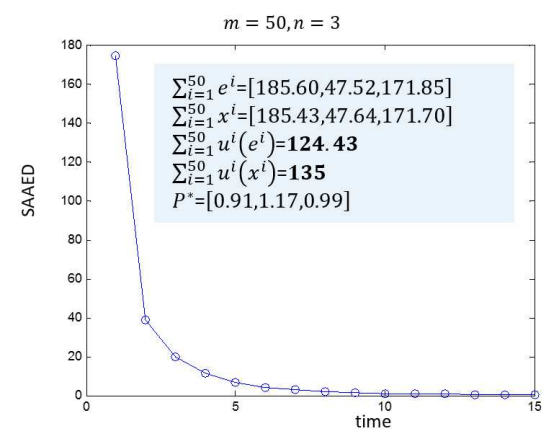

(a) Results on Dataset 1

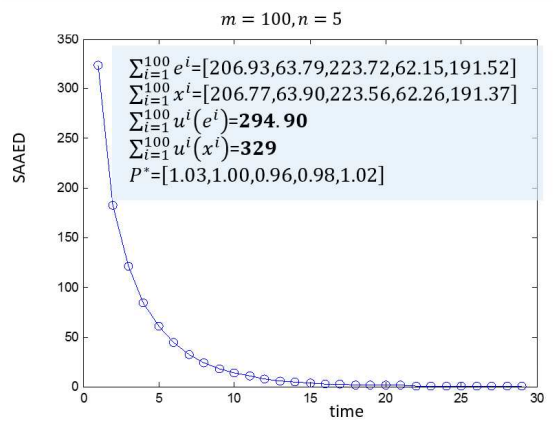

(b) Results on Dataset 2

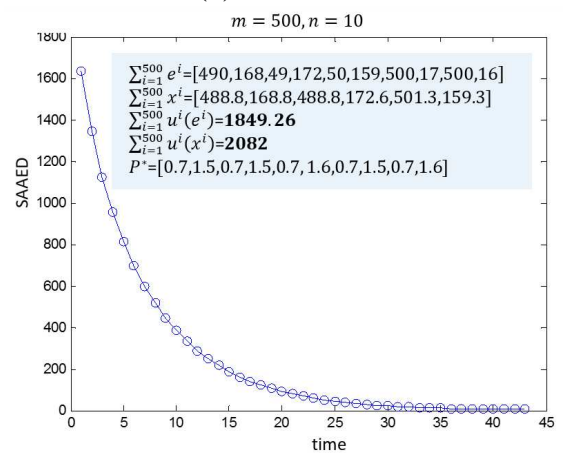

(c) Results on Dataset 3

Fig. 5: The reduction of sum of absolute of aggregate excess demands (SAAED) $\left(\sum_{j=1}^{N}\left|z_{j}\right|\right)$ in subsequent iterations and convergence of price to Walrasian equilibrium.

Lyapunov function $V(\boldsymbol{p})=z_{j}(\boldsymbol{p})$ is proved to be negative and hence the system is asymptotically Lyapunov stable.

\section{Case Study: Pricing and Bandwidth Allocation IN WIRELESS ACCESS NETWORKS}

As a case study, to apply the proposed Walrasian analysis technique, we consider multi-tenant wireless access networks as is the case in HWAN where many wireless network providers (WNPs) share a common radio spectrum. These WNPs offer interchangeable wireless services to the clients. The clients rationally form their request bundles based on their own preference vectors. Figure 6 depicts a sample scenario with three different WNPs.

The pricing of services offered by the WNPs is a central issue to achieve market equilibrium or clean-state. We use our

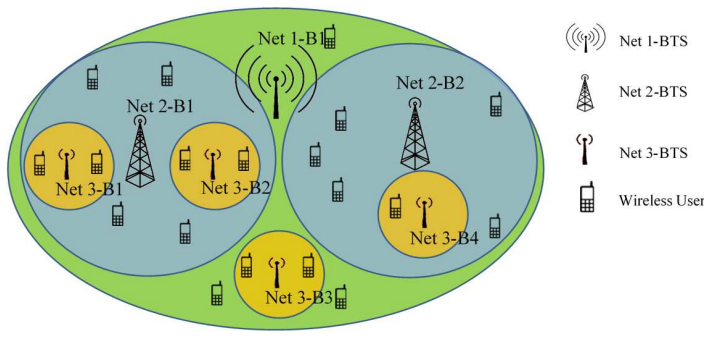

Fig. 6: HWAN environment

Walrasian analysis technique to derive the pricing strategy of the WNPs and as a side product, the final service allocation bundle of the clients are also determined.

For a HWAN with $N$ WNPs and $M$ clients, the overall process is as follows:

- Prepare a Walrasian market for $M$ agents to exchange $N$ commodities;

- Initial asset of agents (e); Each client has an initial asset according to current amount of its commodity; $\mathbf{e}=\left[e_{j}^{i}\right]_{M \times N}$

- Commodities' weight vector (W); Each client assigns a weight for every available commodity in the market; $\mathbf{W}=\left[w_{j}^{i}\right]_{M \times N}$ is an $M \times N$ matrix that $w_{j}^{i}$ indicates the weight of commodity $j$ from the viewpoint of agent $i$;

- Uniform utility function: We use a uniform CES utility function for mathematical tractability (5);

- Demand adjustment: All Clients' adjust their demand vector through an optimization process (1): $x^{i} \leftarrow$ $\chi^{i}(\boldsymbol{p}(t))$

- Evaluating the excess demand: Based on current price vector in time $t$, we calculate the excess demand for each commodity as the difference between requested amount and total available amount of that commodity using the relation (2);

- Price adjustment: To arrive at Walrasian equilibrium price $p^{*}$, the system goes through a price adjustment iterative process. At each iteration $t$, we adjust the price in such a way that the excess demand to be zero. Hence, the price for commodities with positive excess demand should increase and the price for commodities with negative excess demand must decrease.

Here, we assume a HWAN environment with three WNPs and 50 clients, Suppose $\boldsymbol{w}^{i}$ is the weight vector of client $i$. The weight vector of all clients is generated randomly where the average weights for three WNPs are $[0.43,0.41,0.15]$ that means the order of preference of WNPs is $\mathrm{Net}_{1}>\mathrm{Net}_{2}>$ $\mathrm{Net}_{3}$. As a numerical example, we assume WNPs' prepared service is $S=[180,270,340]$ and the initial price $\left(\boldsymbol{p}^{\mathbf{0}}\right)$ for a unit of WNPs service is $5\left(\boldsymbol{p}^{\mathbf{0}}=[5,5,5]\right)$. We are looking for $\mathrm{X}^{*}$ and $\boldsymbol{p}^{*}$.

In Figure 7(a), we show that the sum of absolute of aggregate excess demands (SAAED) $\left(\sum_{j=1}^{N}\left|z_{j}\right|\right)$ that is 7587.67 in first iteration converges to 4.42 in $7^{\prime}$ th it- 


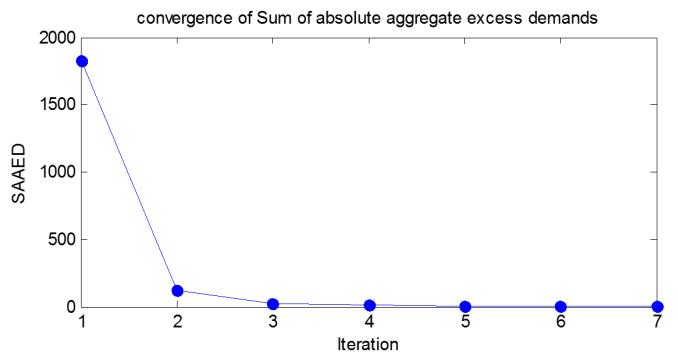

(a) evolution of SAAED $\left(\sum_{j=1}^{N}\left|z_{j}\right|\right)$

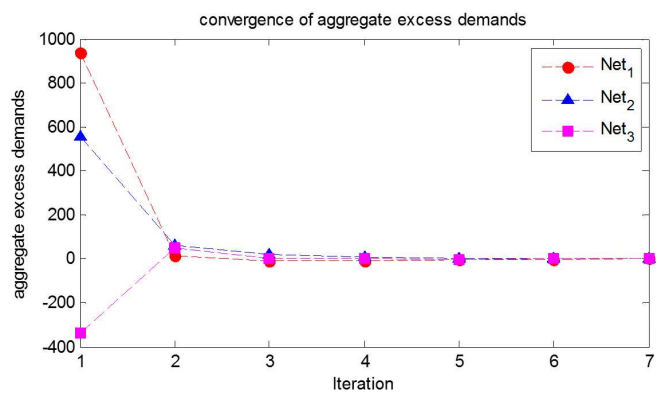

(b) evolution of absolute aggregate excess demands for all commodities

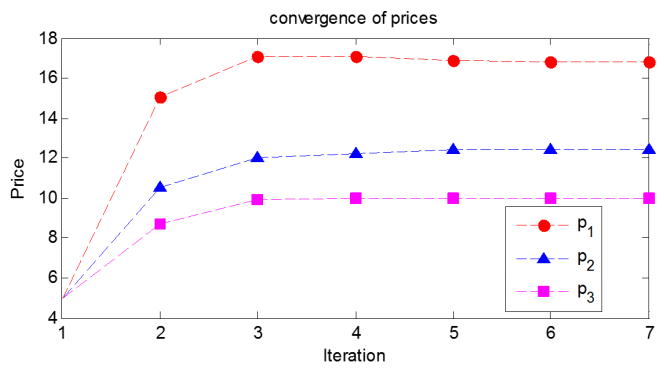

(c) convergence of prices on iterations to $[16.81,12.45,9.98]$

Fig. 7: Evolution of system state (SAAED, excess demand per commodity, price) through analysis iterations.

eration. We defined the condition $\sum_{j=1}^{N}\left|z_{j}\right| \leq 2$ to stop the price adjustment. The values of sum of absolute of aggregate excess demand in the first seven iterations are $[1832.04,121.31,26.62,18.66,4.55,3.63,1.70]$. The aggregate excess demand for all commodities is depicted in Figure 7(b). We see that in the initial stages, there is excess demand for WNPs 1 and 2 and excess supply for WNP 3 because the price of service for WNPs 1 and 2 are low rather than price of WNP 3 and through Walrasian adjustment of prices, the excess demand has dropped to zero. In the last iteration the price vector (Figure 7 (c)) is $\boldsymbol{p}^{*}=[16.81,12.45,9.98]$.

In this case study, clients update their request bundle at the same time and also, WNPs as the commodity moderators adjust their related commodity prices simultaneously. While all decisions are applied in distributed agents, we find that the system rapidly converges to its equilibrium. We have developed a simulation environment using proposed formulations that emulates the behavior of agents (clients and WNPs) through which we could find the Walrasian equilibrium and the best price that cleans the market.

\section{CONCLUSION}

In this paper, we presented a numerical approach for finding the Walrasian equilibrium in exchange economic markets. We modeled the Walrasian analysis engine as a control system with feedback. Our proposed controller, all commodity moderators adjust their prices simultaneously. We showed that over time, the error rate decreases and hence the system converges to its steady state, which is called "cleaning market" state. Based on Walrasian theorem, the steady-state prices reflect the Walrasian equilibrium and the related allocation is Pareto efficient. One of the most suitable fields for using the proposed approach is HWANs where we have WNPs that compete to sell their prepared service. We applied the proposed WAE technique for pricing and bandwidth allocation in an HWAN as a case study and deduced the WNPs' pricing strategy and also determined clients' optimal service bundles. The convergence of the system and the analysis speed was shown to be fast (only four iterations in the scenario with 50 clients) with affordable computational complexity.

\section{REFERENCES}

[1] G. A. Jehle, Advanced microeconomic theory. Pearson Education India, 2001.

[2] G. Debreu, "Valuation equilibrium and pareto optimum," Proceedings of the National Academy of Sciences of the United States of America, vol. 40, no. 7, p. 588, 1954.

[3] — "Market equilibrium," Proceedings of the National Academy of Sciences of the United States of America, vol. 42, no. 11, p. 876, 1956.

[4] T. Negishi, "Welfare economics and existence of an equilibrium for a competitive economy," Metroeconomica, vol. 12, no. 2-3, 1960.

[5] X. Deng, C. Papadimitriou, and S. Safra, "On the complexity of equilibria," in Proceedings of the thiry-fourth annual ACM symposium on Theory of computing. ACM, 2002, pp. 67-71.

[6] F. Echenique and A. Wierman, "Finding a walrasian equilibrium is easy for a fixed number of agents," 2011.

[7] I. S. Wing, "Computable general equilibrium models and their use in economy-wide policy analysis," Joint Program on the Science and Policy of the Global Change, Technical paper, vol. 6, 2004.

[8] S. Mojtaba Matinkhah, S. Khorsandi, and S. Yarahmadian, "A new handoff management system for heterogeneous wireless access networks," International Journal of Communication Systems, vol. 27, no. 7, pp. 1020-1033, 2014.

[9] S. M. Matinkhah, S. Khorsandi, and S. Yarahmadian, "A load balancing system for autonomous connection management in heterogeneous wireless networks," Computer Communications, vol. 97, pp. 111-119, 2017.

[10] V. Haghighatdoost and S. Khorsandi, "Game theoretic spectrum allocation for competing wireless access technologies to maximize the social welfare," Wireless Networks, vol. 25, no. 6, pp. 3557-3577, 2019.

[11] V. Haghighatdoost, S. Khorsandi, and H. Ahmadi, "Fair pricing in heterogeneous internet-of-things wireless access networks using crowdsourcing," IEEE Internet of Things Journal, vol. 8, no. 7, pp. 5710-5721, 2021.

[12] X. Duan, C. Zhao, S. He, P. Cheng, and J. Zhang, "Distributed algorithms to compute walrasian equilibrium in mobile crowdsensing," IEEE Transactions on Industrial Electronics, vol. 64, no. 5, pp. 40484057, 2016.

[13] D. Niyato and E. Hossain, "Spectrum trading in cognitive radio networks: A market-equilibrium-based approach," IEEE Wireless Coтmunications, vol. 15, no. 6, pp. 71-80, 2008.

[14] M. Grant, S. Boyd, and Y. Ye, "Cvx: Matlab software for disciplined convex programming," 2008.

[15] D. Shevitz and B. Paden, "Lyapunov stability theory of nonsmooth systems," IEEE Transactions on automatic control, vol. 39, no. 9, pp. 1910-1914, 1994. 